\section{Routine Procedure Getting Complicated: Iliac Artery Injury during Herniated Disc Surgery}

Sir,

Injuries to iliac vessels are uncommon. These are very rare during routine herniated lumbar disc surgery. If not recognised and corrected at appropriate time, these can be catastrophic. We report a case of such injury, which was successfully managed surgically. This is to sensitise those performing this procedure and to take early action, if needed.

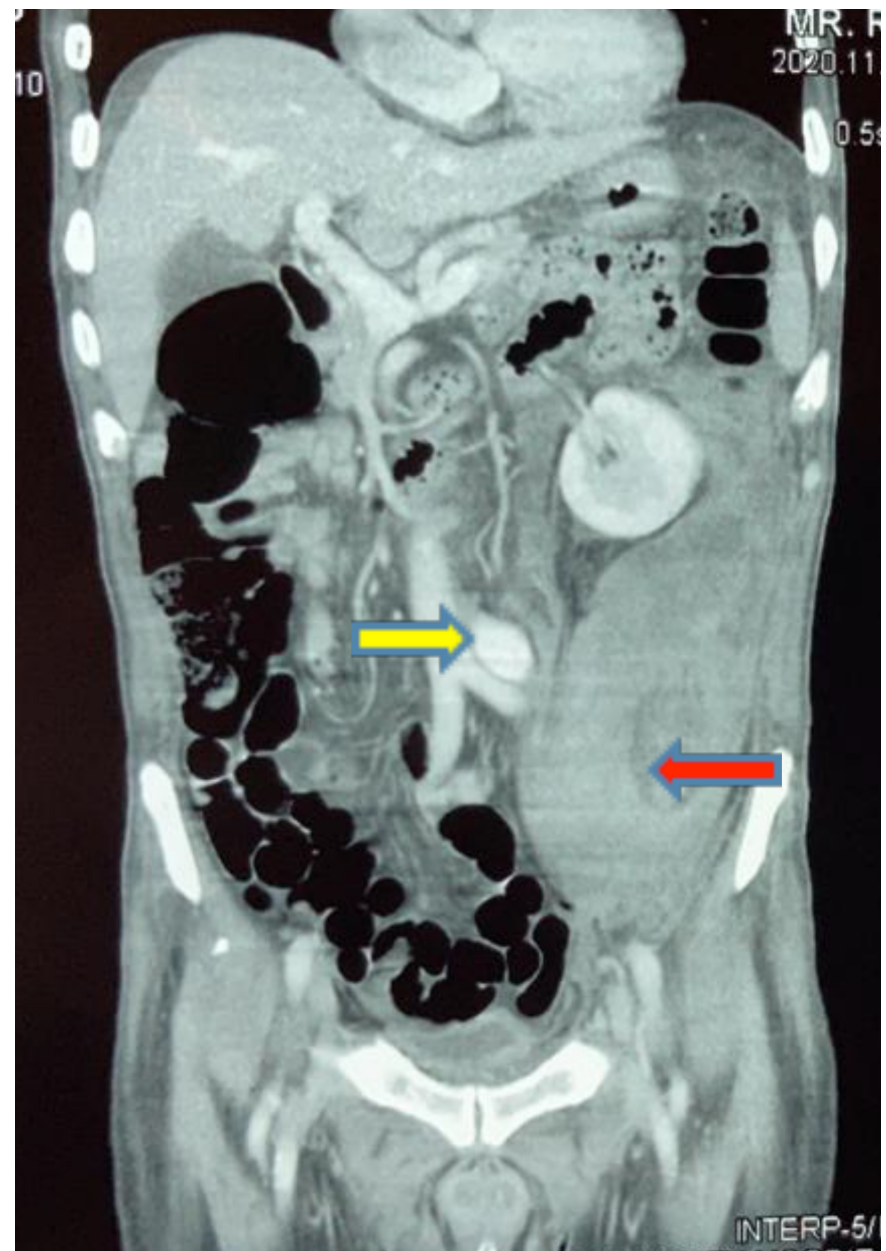

Figure 1: CT abdomen showing large retroperitoneal haematoma (red arrow) and pseudo-aneurysm arising from left common iliac artery (yellowarrow).

A 70-year male had lumbar disc surgery by the posterior approach at another hospital five days ago. He presented in the Emergency Room with abdominal distension and pain. He was pale with cold peripheries and heart rate of 120 beats/min and blood pressure of $70 / 30 \mathrm{mmHg}$. He had distended, mildly tender abdomen. An urgent CT abdomen showed pseudo-aneurysm involving left common iliac artery $(\mathrm{CIA})$ and a large left retroperitoneal haematoma (Figure 1).

The injury was planned to be treated by a covered stent, but diagnostic angiogram showed that the site of left CIA injury was near the aortic bifurcation, thus not suitable for stenting. There was not enough landing zone and higher risk of type 1 endoleak. There was also risk of occluding contralateral iliac arterial lumen by extending the stent into the aorta. Patient was considered for open surgery and was explored through midline, trans-peritoneal approach. Infra-renal aorta, both common iliac arteries and left external and internal iliac arteries were exposed and controlled in a standard fashion. Arterial defect of $1 \times 2 \mathrm{~cm}$ in posterior wall of CIA was noted, which was repaired primarily by prolene $3 / 0$. This helped to control bleeding. Patient made smooth recovery and was discharged on $4^{\text {th }}$ postoperative day.

Injuries to iliac artery are very uncommon during lumbar disc surgery. ${ }^{1}$ They are well protected by anterior longitudinal ligament and retroperitoneal tissue. But injuries have been reported when operations are performed at lower lumber disc levels such as L4-5 or L5-S1. Here, vessels are relatively close to the vertebrae than the proximal segments. They are also at risk of injury when normal anatomical planes are disturbed, such as re-do surgery or anatomical abnormalities of anterior longitudinal ligament or vertebrae. High degree of suspicion and early intervention is key in managing these patients. ${ }^{2}$ Unexpected bleeding from wound or un-explained hypotension during or after the operation should warrant operating team for such injury. Patients need to be treated along the lines of major vascular injuries adhering to the principles of permissive hypotension and keeping the patient warm. ${ }^{3}$ Bleeding from iliacartery injuries either can presentas retroperitoneal haematoma, contained by surrounding retroperitoneal tissue or as active bleeding in peritoneal cavity. The later can have more dramatic presentation and higher mortality. Communication between surgical, anaesthesia and blood bank team is key in successful management of these injuries. If vascular surgery expertise is available in the centre, it should be sought earlier. Midline approach with control of infra-renal aorta and iliac arteries can give enough exposure to localise the injury.

In centres where endovascular intervention facilities are readily available, injury can be treated by placing covered stent. This can avoid laparotomy. In this particular patient, arterial defect was proximally located in left CIA near aortic bifurcation. Hence, it was decided to repair the arterial defect by open surgery.

\section{CONFLICT OF INTEREST:}

The author declared no conflict of interest.

\section{AUTHOR'S CONTRIBUTION:}

ZUR: Study concept, data collection, writing critical review and revision, approval of final draft and responsibilities of all aspect of work. 


\section{REFERENCES}

1. Szolar DH, Preidler KW, Steiner H, Riepl T, Flaschka G, Stiskal M, et al. Vascular complications in lumbar disk surgery: Report of four cases. Neuroradiology 1996; 38(6):521-5. doi: 10.1007/BF00626086

2. Rehman ZU. Peripheral vascular injuries - not to miss or mess them. J Coll Physicians Surg Pak 2019; 29(9):797-8. doi: 10.29271/jcpsp.2019.09.797.

3. Zia Ur Rehman. Abdominal vascular injuries- what general/ trauma surgeons should know. J Pak Med Assoc 2021; 71(8):2027-31. doi: 10.47391/JPMA.354.
Zia Ur Rehman

Section of Vascular Surgery, Department of Surgery, The Aga Khan University Hospital, Karachi, Pakistan

Correspondence to: Dr. Zia Ur Rehman, Section of Vascular Surgery, Department of Surgery, The Aga Khan University Hospital, Karachi, Pakistan

E-mail: ziaur.rehman@aku.edu

Received: March 19, 2021; Revised: May 06, 2021; Accepted: May 06, 2021

DOI: https://doi.org/10.29271/jcpsp.2022.02.272 